\title{
EFFICIENT ANALYSIS OF THE ERGODIC CAPACITY OF COOPERATIVE NON-REgENERATIVE RELAY NETWORKS OVER GENERALIZED FADING CHANNELS
}

\author{
Bhuvan Modi, O. Olabiyi and A. Annamalai \\ Center of Excellence for Communication Systems Technology Research \\ Department of Electrical and Computer Engineering, \\ Prairie View A \& M University, TX 77446 United States of America
}

\begin{abstract}
In this paper, we proposed a novel efficient method of analyzing the ergodic channel capacity of the cooperative amplify-and-forward (CAF) relay system. This is accomplished by employing a very tight approximate moment generating function $(M G F)$ of end-to-end signal-to-noise ratio of 2-hop multi-relay system, which is In this paper, we proposed a novel efficient method of analyzing the ergodic channel capacity of the cooperative amplify-and-forward $(C A F)$ relay system. This is accomplished by employing a very tight approximate moment applicable to myriad of fading environments including mixed and composite fading channels. Three distinct adaptive source transmission policies were considered in our analysis namely: (i) constant power with optimal rate adaptation (ORA); (ii) optimal joint power and rate adaptation (OPRA); and (iii) fixed rate with truncated channel inversion (TCIFR). The proposed frame work based on the novel approximate MGF method is sufficiently general to encapsulate all types of fading environments (especially for the analysis of the mixed fading case)and provides significant advantage to model wireless system for mixed and composite fading channel. In addition to simplifying computation complexity of ergodic capacity for CAF relaying schemes treated in literature, we also derive closed form expressions for the above three adaptive source transmission policies under Nakagami-m fading with i.n.d statistics. The accuracy of our proposed method has been validated with existing MGF expressions that are readily available for specific fading environments in terms of bounds, and via Monte Carlo simulations.
\end{abstract}

\section{KEYWORDS}

Cooperative relay networks, link adaptation, channel capacity, approximate MGF method

\section{INTRODUCTION}

The ever increasing growth in wireless communication services is placing a huge demand for higher data rate, improved network performance and reliability. Several attempts have been made on each of these improvement areas in the literature. One of the earlier contributions is the diversity reception technique, in which multiple reception of transmitted signal over a period of time are combined together to achieve higher received signal-to-noise-ratio and thus improve the system reliability [1]. Another one is the link adaptation, which is also a very powerful wireless

DOI: $10.5121 /$ ijwmn.2016.8503 
communication technique for improving the spectral utilization efficiency. Here, the signaling rate, transmit power, coding rate, constellation size, packet length and so on are "matched" with the prevailing channel conditions, based on the acquired channel side information on the feedback path [2]. If the parameters in the link adaptation belong to different layers in the open system interconnection (OSI) model, then the link adaptation technique is widely regarded as cross-layer design. In the recent times, another major breakthrough in the wireless communication system is the multi-input multiple-output (MIMO), where the transmission diversity has been introduced to improve both the data rate (multiple access application) and reduce transmission error performance (diversity system application). However, the implementation of MIMO techniques in small mobile devices has been greatly limited due to the need to install multiple transmit antenna elements on these small factor devices. More so, most of the existing mobile devices already in use have single antenna and therefore, it would be a daunting task to replace all these devices.

However, this limitation of MIMO implementation on small factor devices has lead to the development of a new communication paradigm known as cooperative relay communication. This novel system takes advantage of the inherent broadcast nature of the wireless transmissions channel to deliver transmitted signal (from a single antenna source) to the destination node (could possess multiple receiver antenna) with the aid of one or more relay nodes to harness a new form of spatial diversity and to combat deleterious effect of multipath fading [3]. Therefore, cooperative communication serves as an alternative to conventional MIMO space diversity technique, as it requires only a single transmit antenna in all communicating nodes, while achieving similar diversity gain as the MIMO system. More so, it is readily implementable on existing mobile handheld devices or wireless sensors. In fact, cooperative diversity is sometimes also referred to as a "distributed MIMO" and it has been shown to provide additional reliability to the wireless system [3]-[4].

In cooperative wireless relay networks, an intermediate relay (i.e., node) may either amplify, what it receives in case of amplify-and-forward relaying protocol or digitally decodes, and reencodes the source information, in case of decode-and-forward relaying protocol before retransmitting it to the destination node [3]. In this paper, we focus on the amplify-and-forward relaying protocol, because it does not require "sophisticated" transceivers at the relays (although our flexible framework is also applicable for digital relaying scheme, once the moment generating function is found or available). While this protocol can achieve a full diversity using a virtual antenna array on each relay nodes, there is a loss of spectral efficiency due to its inherent halfduplex operation. But this penalty could be "recovered" by combining the cooperative diversity with a link adaptation mechanism; wherein the power level, coding rate, constellation size or other transmission parameters are adapted autonomously in response to varying channel conditions. The resulting link adaptive cooperative relay networks could significantly improve the range, rate and reliability of a long-haul inter-cluster communication, thereby improving platform endurance with enhanced low probability of intercept (LPI) and/or low probability of detection (LPD) capability, without using an antenna array. Furthermore, this integration of cooperative wireless communications and link adaptation could be of benefit to the emerging IEEE 802.16 wireless networks (i.e., the current IEEE 802.16e systems employ adaptive modulation, while the emerging IEEE 802.16j standard specifies the use of cooperative diversity in its multi-hop relay architecture).

Although there has been extensive prior research on performance analyses of the adaptive transmission technique for the classical (non-cooperative) wireless networks and the non-adaptive 
cooperative diversity systems, the art of adaptive link layer in cooperative wireless networks is still in its infancy, especially when optimized in a cross-layer design paradigm. For instance, the authors in [2] considered the evaluation of the ergodic capacity of non-cooperative single channel system over fading with different adaptive source transmission schemes; and based on the availability of the channel side information at either the transmitter and/or the receiver. In [5], the author extended this framework to diversity system operating in correlated Rice fading with maximal ratio combining at the destination; yet the considered system is non-cooperative. Also, there have been some contributions on the performance analyses of adaptive transmission on the system capacity of cooperative relay communications. The majority of these literature are limited to unrealistic fading environment (e.g. Rayleigh fading), and fixed signalling rate and/or constant transmit power for all communication nodes. These articles also did not show clearly how the link adaptation (e.g., distributed power control) could be performed with limited channel-side information (CSI), and what are the benefits of jointly optimizing the upper layer protocols with an adaptable physical (PHY) layer? For instance,[6]-[8] have studied the capacity bounds for three-node wireless relay networks in Rayleigh fading environments, but the source rate adaptation [2] was not considered. In [11], the authors studied adaptive transmission for two-hop (regenerative and non-regenerative) relay network (but without the direct source-destination link) in the Rayleigh fading channel. Authors in [12] investigated the channel capacity of SSC diversity in $\eta-\mu$ and $k-\mu$ fading environments, where as in [13] considered the performance of adaptive modulation under generalized selection combining.

However, it is much more realistic to model the channel gain of each link in a network as a Rice or Nakagami-m random variable, due to the increased likelihood of the presence of a strong specular component in an airborne platform. It has been shown in the literature that the evaluation of the moment generating function (MGF), probability density function (PDF) and the cumulative distribution function (CDF) of harmonic mean of two-hop relayed path is a daunting task with existing results, limited to the Rayleigh fading [12] and the Nakagami-m [13] fading channel with integer $m$ and even in such cases the expressions are too complicated; and not useful for system level analysis. However, it has been shown in [14] that the harmonic mean SNR, $\gamma_{i}^{(H M)}$ in (1) can effectively approximate $\gamma_{i}$ especially at medium and high SNR. Also, in this case the MGF expressions are still difficult to obtain with existing results limited to the Raleigh fading [15] and i.i.d. Nakagami-m [14] channels. Due to this limitation, bounds have been developed for two hoprelayed paths. The authors in [16] derived bounds for the ergodic capacity analysis of a linkadaptive cooperative non-regenerative relay system with limited channel side information (CSI). However, their analysis is limited to the Rayleigh fading. Moreover, motivated by [16], the authors in [17] extended the work of conventional cooperative-diversity networks in [16] to relayselection based cooperative diversity networks with adaptive source transmissions. Yet, their analysis is limited to the Rayleigh fading channel. Authors in [18] subsequently extended the framework developed in [16] to decode-and-forward relay networks. In [19] the authors studied the effect of source transmission adaptation on capacity of multi relay 2-hop CAF relay system over generalized fading channel, but the presented results are limited to independent and identically distributed (i.i.d) fading channel. Whereas authors in [20-22] extensively studied the effect of source transmission adaptation on capacity of 2-hop CAF relay system by developing MGF and CDF approaches that facilitates the evaluation of the ergodic capacity of cooperative relay system in fading environments. Also author in [23] derived the MGF approach for CAF network with adaptive modulation in Nakagami-m fading environments. However, because the MGF expressions ( for independent and non-identically distributed (i.n.d) Nakagami-m and Rice fading channels ) used in their numerical analysis is derived from loose bounds of the end-to-end 
SNR, the accuracy of their observation can might not be satisfactory under certain condition. This is because of the propagation of errors coming from the MGF of bound of end-to-end SNR.

Motivated by the above mentioned discussions/observations, in this paper we seek to present considerably simpler analytical framework for efficient computation of the ergodic channel capacities of dual-hop CAF relay networks under ORA, OPRA and TCIFR source-adaptive transmission policies in a myriad of fading environments. The key contributions of this paper are summarized below:

(i) The significant contribution of this paper is, we bring together a flexible and highly accurate approximate MGF expression that has been shown to be much more accurate and straightforward to compute than the MGF bounds employed in [16], [20]. It is interesting to note that this efficient MGF expression does not only reduce the computational complexity for evaluating the ergodic capacity for common fading channel, the expression is sufficiently general and straightforward to represent composite and even mixed fading environments (i.e. see fig. 7, which shows the scenario with combination of Rice, Nakagami and Rayleigh fading channels on one system model). This facilitates the efficient and accurate evaluation of the ergodic capacity of link adaptive CAF relay networks with three distinct source adaptive transmission policies: (i) ORA; (ii) OPRA; and (iii) TCIFR, over the wide range of fading environments for the non-identically distributed (i.n.d) fading statistics. Also, the proposed framework allows us to gain insights in to how fade distributions and dissimilar fading statistics across the distinct communication links affect the ergodic capacity, without imposing any restrictions on the fading parameters. (Details expression is presented in section 2.1)

The efficacy of our proposed methodology is further demonstrated in the need for closedform MGF expression for intuitive understanding of the parameters affecting the ergodic capacity of ORA, OPRA and TCIFR source transmission schemes. Here, we decompose the MGF of end-to-end SNR of the single CAF relay system to obtain new forms of MGF and CDF expressions that enables us to come up with closed form expression for the ergodic capacity under the three adaptive source transmission policies. However, it is worth to mention that, the partial expansion decomposition is only applicable for the single CAF relay over Nakagami-m (with integer $m$ values) and more tedious for the multiple relay system. Therefore, from the computational efficiency perspective, the ergodic capacity of higher diversity order with non-integer $m$ is considerably simpler to compute numerically using our approximate closed-form MGF (in conjunction with Fixed-Talbot method [24]). (Detail expression is presented in section 3)

Note: This paper is extension with some significant improvement in results presented in our paper [20], where [20] presents ergodic capacity analysis of CAF relay network for MGF of upper and lower bound over Rice and Nakagami-m fading channel. In this paper we are utilizing "approximate MGF" to derive ergodic capacity analysis for wide range of fading channels (ie., these results not only restricted to Rayleigh or Nakagami fading channel but it covers wide range of fading channels and mixed fading case as well by simply introducing MGF of single channel as explained in (i) and also derives closed form expression for the ergodic capacity over Nakagami-m fading channel under the three adaptive source transmission policies as explained in (ii). 
The remainder of this paper is organized as follows. In section 2, the system model is discussed. The capacity analysis for the cooperative relay networks under three distinct adaptive transmission techniques is presented in section 3. Selected numerical results are presented in section 4. Our conclusions are given in section 5.

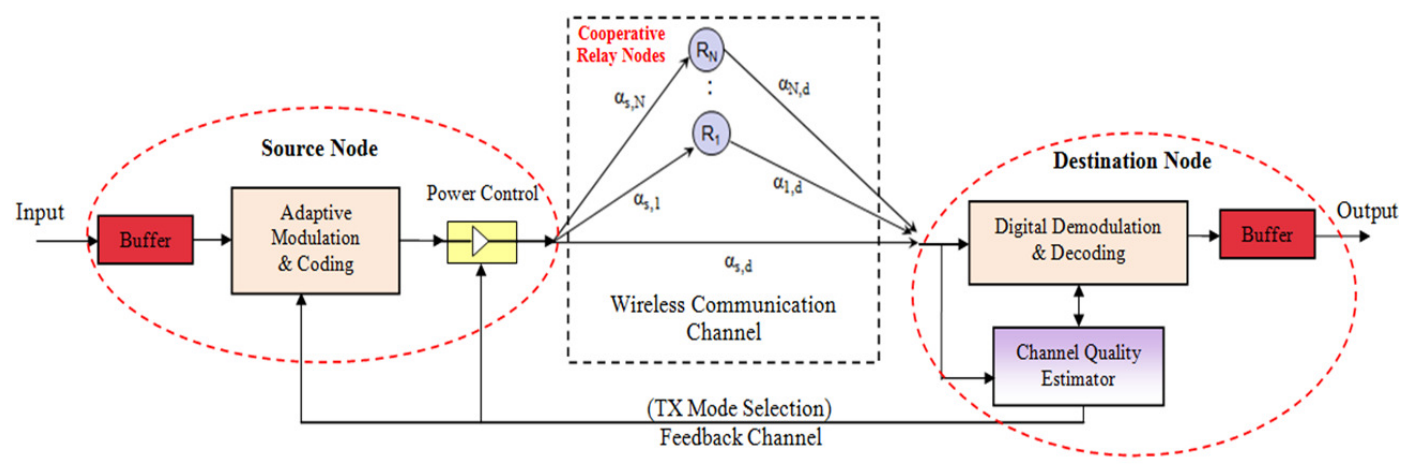

Figure 1. Link-adaptive cooperative relay networks

\section{System Model}

Figure 1 illustrates the link-adaptive cooperative wireless system, consisting of a source node $S$ communicates with a destination node $D$ via a direct link and through $N$ amplify-and-forward relays $R_{i}, i \in\{1,2, \ldots, N\}$, in two transmission phases. In the first phase of cooperation, node $\mathrm{S}$ transmits the signal $x$ to the $\mathrm{D}$ and the relays $R_{i}$, where the channel fading coefficients between $S$ and $D, S$ and the $i$-th relay node $R_{i}$; and $R_{i}$ and $D$ are denoted by $\alpha_{s, d}, \alpha_{s, i}$ and $\alpha_{i, d}$ respectively. During the second phase of cooperation, orthogonal transmission is required to transmit the received signals at each of the $N$ relays; this can be accomplished by using TDMA in a roundrobin fashion and/or FDMA. If the $i^{\text {th }}$ amplifier gain is chosen as $G_{i}=\sqrt{E_{s} /\left(E_{s}\left|\alpha_{s i}\right|^{2}+N_{0}\right)}$ (where $E_{s}$ denotes the average symbol energy and $N_{0}$ corresponds to the noise variance) and maximal-ratio combiner (MRC) is implemented at node $D$ to coherently combine all the signals received during the two transmission phases, the total received SNR at output of the MRC detector can be shown to be [3], [25]

$$
\gamma=\gamma_{s, d}+\sum_{i=1}^{N} \frac{\gamma_{s, i} \gamma_{i, d}}{1+\gamma_{s, i}+\gamma_{i, d}}=\gamma_{s, d}+\sum_{i=1}^{N} \gamma_{i} \leq \gamma_{s, d}+\sum_{i=1}^{N} \gamma_{i}^{(H M)}
$$

where $\gamma_{i}^{(H M)}=\frac{\gamma_{s, i} \gamma_{i, d}}{\gamma_{s, i}+\gamma_{i, d}}$ is the harmonic mean SNR and $\gamma_{a, b}=\left|\alpha_{a, b}\right|^{2} E_{s} / N_{o}$ corresponds to the instantaneous SNRs of link $a-b$.

\subsection{Approximate MGF of end-to-end SNR for Generalized Fading Channels (Especially Mixed Fading Channels).}

In this section we will present the approximate MGF expression, which is simple and can handle complex and mixed fading channel models. Using the upper bound of SNR in ([20], eq. (3)), the cumulative distribution function (CDF) of the relayed path can be approximat 


$$
F_{\gamma_{i}}^{(U B)}(\gamma)=1-\left[1-F_{\gamma_{s, i}}(\gamma)\right]\left[1-F_{\gamma_{i, d}}(\gamma)\right]
$$

where $F_{\gamma_{s, i}}(\gamma)$ and $F_{\gamma_{i, d}}(\gamma)$ are the CDFs of the source-to-relay and relay-to-destination links, respectively. The MGF of $\gamma_{i}$ can be determined by differentiating the right hand side of (2) with respect to $\gamma$, and then evaluating the Laplace transform of the resulting expression, viz.,

$$
\phi_{\gamma_{i}}^{(U B)}(s)=\phi_{\gamma_{s, i}}(s)+\phi_{\gamma_{i, d}}(s)-\int_{0}^{\infty} e^{-s \gamma}\left[f_{\gamma_{s, i}}(\gamma) F_{\gamma_{i, d}}(\gamma)+f_{\gamma_{i, d}}(\gamma) F_{\gamma_{s, i}}(\gamma)\right] d \gamma
$$

Interestingly, the last term of (3) resembles the MGF of output SNR for a dual-diversity selection combining, and therefore (3) can be re-stated as

$$
\phi_{\gamma_{i}}^{(U B)}(s)=\phi_{\gamma_{s, i}}(s)+\phi_{\gamma_{i, d}}(s)-\phi_{\gamma_{S D C}}(s)
$$

where $\phi_{\gamma_{a, b}}(s)$ is the MGF of a single channel for link $a$ to $b$. Eq. (3) and/or (4) are the typical MGF bounds that have been used for cooperative amplify-and-forwarded (CAF) relay networks in [20]. However the resulting expressions for the Nakagami-m, Rice and mixed-fading channels can be quite complicated especially. But recognizing that $\phi_{\gamma_{S D C}}(s)>\phi_{\gamma_{M R C}}(s)$, we can further approximate (3) as [26]

$$
\phi_{\gamma_{i}}^{(A)}(s) \approx \phi_{\gamma_{s, i}}(s)+\phi_{\gamma_{i, d}}(s)-\phi_{\gamma_{s, i}}(s) \phi_{\gamma_{i, d}}(s)
$$

since $\phi_{\gamma_{M R C}}(s)=\phi_{\gamma_{s, i}}(s) \phi_{\gamma_{i, d}}(s)$. Eq. (5) greatly reduces the computational burden as it involves only the MGF of SNR for single channel reception (i.e., it is represented in terms of MGF between $S$ to relay $i$ and relay $i$ to destination $D$ ). Moreover, it has already been shown in [23] that (5) accurately approximates (4) over a myriad of wireless fading channels. This expression allows us to simplify the computation complexity of the performance metrics in the CAF relay system under generalized fading environments. It is also interesting to note that, the MGF expression in (5) is simple and can readily handle complex (e.g. Suzuki distribution) and mixed fading channel models (which is daunting task with exact and bounding techniques in the existing propositions).

\subsection{Cumulative Distribution Function (CDF) of the Total Effective SNR.}

As we will show in the next section, the knowledge of the CDF of the total effective SNR $\gamma$ of the CAF relay networks is required for the evaluation of OPRA and TCIFR capacity. Since the analytical CDF expression for the CAF relay networks is difficult to obtain, the alternative is to compute the CDF from the MGF expression in [20, eq. (2)] (i.e., $\phi_{\gamma}(s)=\phi_{\gamma_{s, d}}(s) \prod_{i=1}^{N} \phi_{\gamma_{i}}(s)$, where $\phi_{\gamma_{s, d}}(s)$ is the MGF between source to destination of appropriate fading channel and $\phi_{r_{i}}(s)$ is the MGF of a two hop-relayed path). One of the most efficient frequency inversion method is the Abate's Fixed-Talbot method, (i.e., multi-precision Laplace transform inversion) [24], viz., 


$$
F_{X}(x) \cong \frac{1}{2 Z} \phi_{X}(r) e^{r x}+\frac{r}{Z} \sum_{k=1}^{Z-1} \operatorname{Re}\left\{\frac{1+j \sigma\left(\theta_{k}\right)}{s\left(\theta_{k}\right)} e^{x s\left(\theta_{k}\right)} \phi_{X}\left(s\left(\theta_{k}\right)\right)\right\}
$$

where $r=2 Z /(5 x), \theta_{k}=k \pi / Z, \sigma\left(\theta_{k}\right)=\theta_{k}+\left(\theta_{k} \cot \left(\theta_{k}\right)-1\right) \cot \left(\theta_{k}\right), \quad s\left(\theta_{k}\right)=r \theta_{k}\left(j+\cot \left(\theta_{k}\right)\right)$, and a positive integer $Z$ can be chosen to get the desired accuracy.

\section{Ergodic Capacity Computation in Fading Channels}

In this section, we will derive closed-form expressions for the ergodic capacity under three adaptive source transmission policies.

\subsection{Optimal Rate Adaptation with Fixed Transmit Power (ORA)}

When only the rate is adapted by changing channel conditions, the ergodic capacity is given by [20]

$$
\frac{\bar{C}_{O R A}}{B}=\frac{1}{N+1} \frac{1}{\ln 2} \int_{0}^{\infty} \frac{e^{-y}}{y}\left[1-\phi_{\gamma}(y)\right] d y
$$

where $N$ is the number of relays and $B$ is the channel bandwidth.

Expression (7) indicates that the ORA capacity evaluation requires only the knowledge of the MGF of SNR of fading channel. For arbitrary fading environment, the integral can be evaluated using numerical method. However, closed-form solutions can be obtained for special cases. For instance, following the procedure in Appendix A by applying the auxiliary equation (B.7) in conjunction with the decomposed PDF expression obtain via (A.2), the closed-form ORA capacity for single CAF relay system with Nakagami-m fading can be expressed as

$$
\begin{aligned}
\frac{\bar{C}_{O R A}}{B}= & \frac{1}{2} \log _{2}(e)\left\{\sum_{p=1}^{m_{s, d}} \alpha_{p} e^{-m_{s, d} / \Omega_{s, d}} \sum_{b=1}^{p}\left(\frac{m_{s, d}}{\Omega_{s, d}}\right)^{p-b} \Gamma\left(-b+p, \frac{m_{s, d}}{\Omega_{s, d}}\right)+\sum_{q=1}^{m_{s, i}} \alpha_{q} e^{-m_{s, i} / \Omega_{s, i}} \sum_{b=1}^{q}\left(\frac{m_{s, i}}{\Omega_{s, i}}\right)^{q-b} \Gamma\left(-b+q, \frac{m_{s, i}}{\Omega_{s, i}}\right)\right. \\
& +\sum_{r=1}^{m_{i, d}} \alpha_{r} e^{-m_{i, d} / \Omega_{i, d}} \sum_{b=1}^{r}\left(\frac{\Omega_{i, d}}{m_{i, d}}\right)^{r-b} \Gamma\left(-b+r, \frac{m_{i, d}}{\Omega_{i, d}}\right)+\sum_{w=1}^{m_{s, d}} \alpha_{w} e^{-m_{s, d} / \Omega_{s, d}} \sum_{b=1}^{w}\left(\frac{m_{s, d}}{\Omega_{s, d}}\right)^{w-b} \Gamma\left(-b+w, \frac{m_{s, d}}{\Omega_{s, d}}\right) \\
& -\sum_{t=1}^{m_{s, d}} \alpha_{t} e^{-m_{s, d} / \Omega_{s, d}} \sum_{b=1}^{t}\left(\frac{m_{s, d}}{\Omega_{s, d}}\right)^{t-b} \Gamma\left(-b+t, \frac{m_{s, d}}{\Omega_{s, d}}\right)-\sum_{u=1}^{m_{s, i}} \alpha_{u} e^{-m_{s, i} / \Omega_{s, i}} \sum_{b=1}^{u}\left(\frac{m_{s, i}}{\Omega_{s, i}}\right)^{u-b} \Gamma\left(-b+u, \frac{m_{s, i}}{\Omega_{s, i}}\right) \\
& \left.-\sum_{v=1}^{m_{i, d}} \alpha_{v} e^{-m_{i, d} / \Omega_{i, d}} \sum_{b=1}^{v}\left(\frac{\Omega_{i, d}}{m_{i, d}}\right)^{v-b} \Gamma\left(-b+v, \frac{m_{i, d}}{\Omega_{i, d}}\right)\right\}
\end{aligned}
$$

where $\Omega_{a, b}=E\left[\gamma_{a, b}\right]$ denotes the mean link SNR, $m_{a, b}$ denotes the Nakagami-m fading severity index, $\Gamma(.,$.$) denotes the upper incomplete Gamma function and the coefficients \alpha_{p}, \alpha_{q}, \alpha_{r}, \alpha_{w}$, $\alpha_{t}, \alpha_{u}$ and $\alpha_{v}$ are defined in (A.3).

\subsection{Optimal Power and Rate Adaptation (OPRA)}

In the case of OPRA strategy, both the transmission power and rate are matched to the varying channel conditions through use of a multiplexed multiple codebook design. This leads to the highest achievable capacity with CSI. From [20], we have 


$$
\frac{\bar{C}_{O P R A}}{B}=\frac{1}{N+1} \frac{1}{\ln 2} \int_{0}^{\infty} \frac{1}{x}\left[e^{-2 x}\left[1-F_{\gamma}\left(\gamma_{0}\right)\right]-\phi_{\gamma}\left(\frac{2 x}{\gamma_{0}}\right)+F_{\hat{\gamma}}\left(\gamma_{0}\right)\right] d x
$$

If $\phi_{\gamma}\left(2 x / \gamma_{0}\right)$ is available in the closed form, the CDF terms, $F_{\gamma}\left(\gamma_{0}\right)$ and $F_{\gamma}\left(\gamma_{0}\right)$ in (9) can be computed efficiently using (6) and $\gamma_{0}$ is the optimal cut-off SNR below which the data transmission is suspended.

To achieve the capacity (9), the channel fade level (i.e., CSI) tracked at the receiver must be conveyed to the transmitter on the feedback path for power and rate adaptation in accordance with the time-varying nature of the channel fading. When $\gamma<\gamma_{0}$, no data is transmitted, and thus the optimal policy suffers an outage probability given by $P_{\text {out }}=F_{\gamma}\left(\gamma_{0}\right)=1-F_{\gamma}^{c}\left(\gamma_{0}\right)$, which equals the probability of no transmission. The optimal cutoff SNR must satisfy

$$
F_{\gamma}^{c}\left(\gamma_{0}\right)-\gamma_{0}[1-\zeta]=0
$$

where $\zeta=\frac{1}{\pi} \int_{0}^{\infty} \operatorname{Re}\left\{\phi_{\gamma}(-j \omega) E i\left(-j \omega \gamma_{0}\right)\right\} d \omega$ and the exponential integral $E i(-j c)=-\int_{1}^{\infty} \frac{e^{-j c t}}{t} d t$. Whereas $\gamma_{0}$ can be determined by solving (10) numerically.

Similar to the capacity of ORA scheme, closed-form solution for (9) can be obtained for single CAF relay with Nakagami-m channel using Appendix B by combining the auxiliary result in (B.12) and the end-to-end PDF expression obtain via (A.2) which is given by

$$
\begin{aligned}
\frac{\bar{C}_{\text {OPRA }}}{B}= & \frac{1}{2} \log _{2}(e)\left\{\sum_{p=1}^{m_{s, d}} \alpha_{p} \sum_{b=0}^{p-1} \frac{\Gamma\left(b, \gamma_{0} \frac{m_{s, d}}{\Omega_{s, d}}\right)}{b !}+\sum_{q=1}^{m_{s, j}} \alpha_{q} \sum_{b=0}^{g-1} \frac{\Gamma\left(b, \gamma_{0} \frac{m_{s, i}}{\Omega_{s, i}}\right)}{b !}+\sum_{r=1}^{m_{s, d}} \alpha_{r} \sum_{b=0}^{r-1} \frac{\Gamma\left(b, \gamma_{0} \frac{m_{s, d}}{\Omega_{s, d}}\right)}{b !}\right. \\
& \left.+\sum_{n=1}^{m_{i, d}} \alpha_{w} \sum_{b=0}^{n-1} \frac{\Gamma\left(b, \gamma_{0} \frac{m_{i, d}}{\Omega_{i, d}}\right)}{b !}-\sum_{t=1}^{m_{s, d}} \alpha_{t} \sum_{b=0}^{t-1} \frac{\Gamma\left(b, \gamma_{0} \frac{m_{s, d}}{\Omega_{s, d}}\right)}{b !}-\sum_{u=1}^{m_{s, i}} \alpha_{u} \sum_{b=0}^{u-1} \frac{\Gamma\left(b, \gamma_{0} \frac{m_{s, i}}{\Omega_{s, i}}\right)}{b !}-\sum_{v=1}^{m_{s, d}} \alpha_{v} \sum_{b=0}^{v-1} \frac{\Gamma\left(b, \gamma_{0} \frac{m_{t, d}}{\Omega_{l, d}}\right)}{b !}\right\}
\end{aligned}
$$

\subsection{Channel Inversion with Fixed Rate (CIFR)}

In CIFR policy, the transmitter adapts its power, based on channel fading to maintain a constant received SNR at the receiver, and uses fixed-rate modulation and fixed-code designs. This technique is the least complex to implement given that the reliable channel estimates are available at the transmitter. However, when the channel experiences deep fades, the penalty in transmit power requirement with the CIFR policy will be enormous, because channel inversion needs to compensate for deep fades. To overcome this, a modified inversion policy, truncated channel inversion and fixed rate policy (TCIFR) was considered in [2], where the channel fading is only inverted above a fixed cutoff fade depth $\gamma_{0}$. The data transmission is ceased if $\gamma$ falls below 
optimal cutoff $\operatorname{SNR} \gamma_{0}$. In this case, it is easy to show that the outage probability is $P_{\text {out }}=F_{\gamma}\left(\gamma_{0}\right)=1-F_{\gamma}^{c}\left(\gamma_{0}\right)$ and the channel capacity is given by [20]

$$
\frac{\bar{C}_{T C I F R}}{B}=\frac{1}{N+1} \log _{2}\left(1-\frac{1}{\nabla}\right) F_{\gamma}^{c}\left(\gamma_{0}\right)
$$

where $\nabla=\frac{1}{\pi} \int_{0}^{\infty} \operatorname{Re}\left\{\phi_{\gamma}(-j \omega) E i\left(-j \omega \gamma_{0}\right)\right\} d \omega$. Observe that, if the MGF of $\gamma$ is known in closed-form, then the above integral can be evaluated efficiently via Gauss-Chebyshev Quadrature method for a wide-range of fading channel models and diversity combining techniques employed (including maximal-ratio combining and selection diversity). The zero-outage ergodic capacity (i.e., CIFR policy) can be evaluated by setting $\gamma_{0}=0$ in (10).

Now, for the special case of single CAF relay with Nakagami-m channel, we can obtain closedform expressions for $\nabla$ and $F_{\gamma}^{c}\left(\gamma_{0}\right)$ in (12) using the procedure in Appendix B and are given respectively by

$$
\begin{aligned}
& \nabla=\left\{\sum_{p=1}^{m_{s, d}} \alpha_{p} \frac{\Gamma\left(p-1, \gamma_{0} \frac{m_{s, d}}{\Omega_{s, d}}\right)}{\frac{\Omega_{s, d}}{m_{s, d}} \Gamma(p)}+\sum_{q=1}^{m_{s, i}} \alpha_{q} \frac{\Gamma\left(q-1, \gamma_{0} \frac{m_{s, i}}{\Omega_{s, i}}\right)}{\frac{\Omega_{s, i}}{m_{s, i}} \Gamma(q)}+\sum_{r=1}^{m_{s, d}} \alpha_{r} \frac{\Gamma\left(r-1, \gamma_{0} \frac{m_{s, d}}{\Omega_{s, d}}\right)}{\frac{\Omega_{s, d}}{m_{s, d}} \Gamma(r)}+\right. \\
& \left.\sum_{w=1}^{m_{i, d}} \alpha_{w} \frac{\Gamma\left(w-1, \gamma_{0} \frac{m_{i, d}}{\Omega_{i, d}}\right)}{\frac{\Omega_{i, d}}{m_{i, d}} \Gamma(w)}-\sum_{t=1}^{m_{s, d}} \alpha_{t} \frac{\Gamma\left(t-1, \gamma_{0} \frac{m_{s, d}}{\Omega_{s, d}}\right)}{\frac{\Omega_{s, d}}{m_{s, d}} \Gamma(t)}-\sum_{u=1}^{m_{s, i}} \alpha_{u} \frac{\Gamma\left(u-1, \gamma_{0} \frac{m_{s, i}}{\Omega_{s, i}}\right)}{\frac{\Omega_{s, i}}{m_{s, i}} \Gamma(u)}-\sum_{v=1}^{m_{i, d}} \alpha_{v} \frac{\Gamma\left(v-1, \gamma_{0} \frac{m_{i, d}}{\Omega_{i, d}}\right)}{\frac{\Omega_{i, d}}{m_{i, d}} \Gamma(v)}\right\}
\end{aligned}
$$

and

$$
\begin{aligned}
& F_{\gamma}^{c}\left(\gamma_{0}\right)=\left\{\sum_{p=1}^{m_{s, d}} \alpha_{p} \frac{\Gamma\left(p, \gamma_{0} \frac{m_{s, d}}{\Omega_{s, d}}\right)}{\Gamma(p)}+\sum_{q=1}^{m_{s, i}} \alpha_{q} \frac{\Gamma\left(q, \gamma_{0} \frac{m_{s, i}}{\Omega_{s, i}}\right)}{\Gamma(q)} \sum_{r=1}^{m_{s, d}} \alpha_{r} \frac{\Gamma\left(r, \gamma_{0} \frac{m_{s, d}}{\Omega_{s, d}}\right)}{\Gamma(r)}+\sum_{w=1}^{m_{i, d}} \alpha_{w} \frac{\Gamma\left(w, \gamma_{0} \frac{m_{i, d}}{\Omega_{i, d}}\right)}{\Gamma(w)}\right. \\
& \left.-\sum_{t=1}^{m_{s, d}} \alpha_{t} \frac{\Gamma\left(t, \gamma_{0} \frac{m_{s, d}}{\Omega_{s, d}}\right)}{\Gamma(t)}-\sum_{u=1}^{m_{s, i}} \alpha_{u} \frac{\Gamma\left(u, \gamma_{0} \frac{m_{s, i}}{\Omega_{s, i}}\right)}{\Gamma(u)}-\sum_{v=1}^{m_{i, d}} \alpha_{v} \frac{\Gamma\left(v, \gamma_{0} \frac{m_{i, d}}{\Omega_{i, d}}\right)}{\Gamma(v)}\right\}
\end{aligned}
$$

\section{NUMERICAL RESULTS}

In this section, we will discuss the efficacy of our analytical frameworks based on the approximate MGF method (i.e., $\left.\phi_{\gamma}^{(A)}(s)\right)$ by comparing it with bounding techniques (i.e., $\left.\gamma_{i}^{(L B)}=\frac{1}{2} \min \left(\gamma_{s, i}, \gamma_{i, d}\right) \leq \gamma_{i} \leq \gamma_{i}^{(U B)}=\min \left(\gamma_{s, i}, \gamma_{i, d}\right)[20]\right)$, and simulation for the ergodic capacity analysis of cooperative analog relaying systems under three distinct source adaptive transmission 
policies. The following mean link SNRs (arbitrarily chosen) will be used to generate the plots, unless stated otherwise: $\Omega_{s, 1}=E_{s} / N_{0}, \Omega_{s, 2}=0.5 E_{s} / N_{0}, \Omega_{1, d}=0.5 E_{s} / N_{0}, \Omega_{2, d}=E_{s} / N_{0}$, and $\Omega_{s, d}=$ $0.2 E_{s} / N_{0}$.

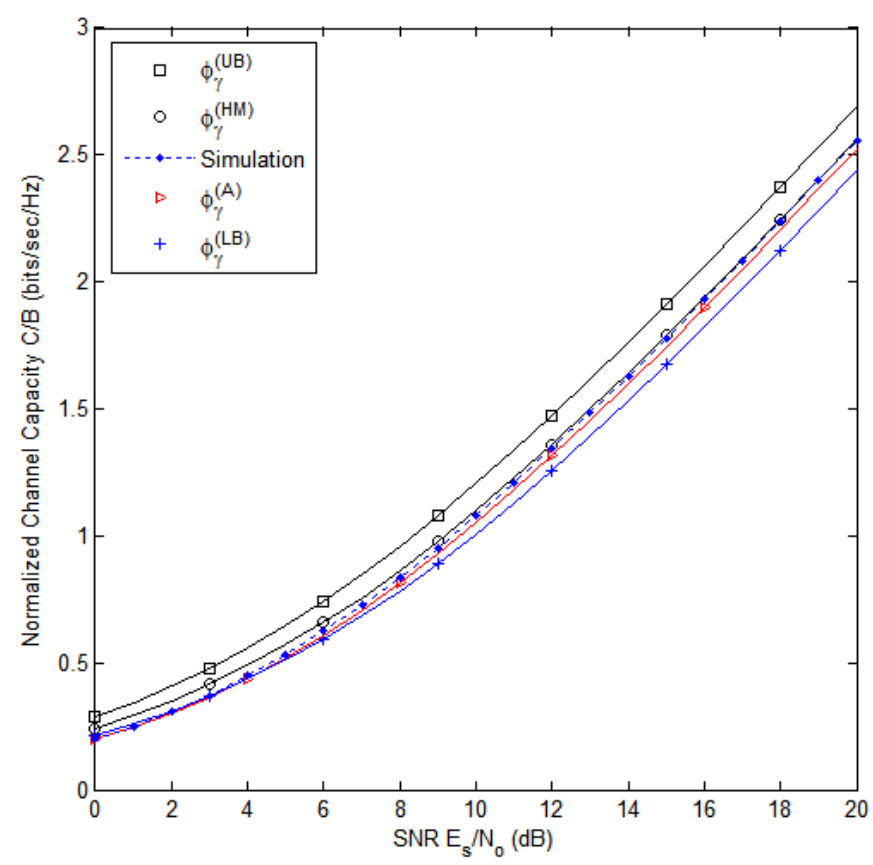

Figure 2. Ergodic capacities of a cooperative relay network with ORA policy in Rayleigh fading channels with single relay diversity $(N=1)$.

\section{Example 1: Rayleigh fading channels}

Fig. 1 shows the ergodic capacity analysis of a CAF relay network with ORA policy under i.n.d Rayleigh fading channels, using our proposed approximate MGF $\phi_{\gamma}^{(A)}($.) method. For the sake of comparison, we also presented plots using bounds (i.e., Upper bound MGF $\phi_{\gamma}^{(U B)}(s)$ or Lower Bound $\operatorname{MGF}_{\gamma}^{(L B)}(s)$ [16]), harmonic mean bound [15]

$$
\phi_{\eta_{i}}^{(H M)}(s)=\left[\left(1 / \Omega_{s, i}-1 / \Omega_{i, d}\right)^{2}+\left(1 / \Omega_{s, i}+1 / \Omega_{i, d}\right) s\right] / \Delta^{2}+\frac{2 s}{\Delta^{3} \Omega_{s, i} \Omega_{i, d}} \ln \left(\left(s+\Delta+\frac{1}{\Omega_{s, i}}+\frac{1}{\Omega_{i, d}}\right)^{2} \frac{\Omega_{s, i} \Omega_{i, d}}{4}\right)
$$

and the simulation. It is interesting to note that both bounds enclose the "exact" (simulation) and the approximation $\phi_{\gamma}^{(A)}($.) with all the curves following the expected trend. It is also worth mentioning that the approximation is much closer to the simulation. Therefore, introducing the "approximate MGF" allows us to further simplify the computation of the ergodic capacity of the CAF relay system. 


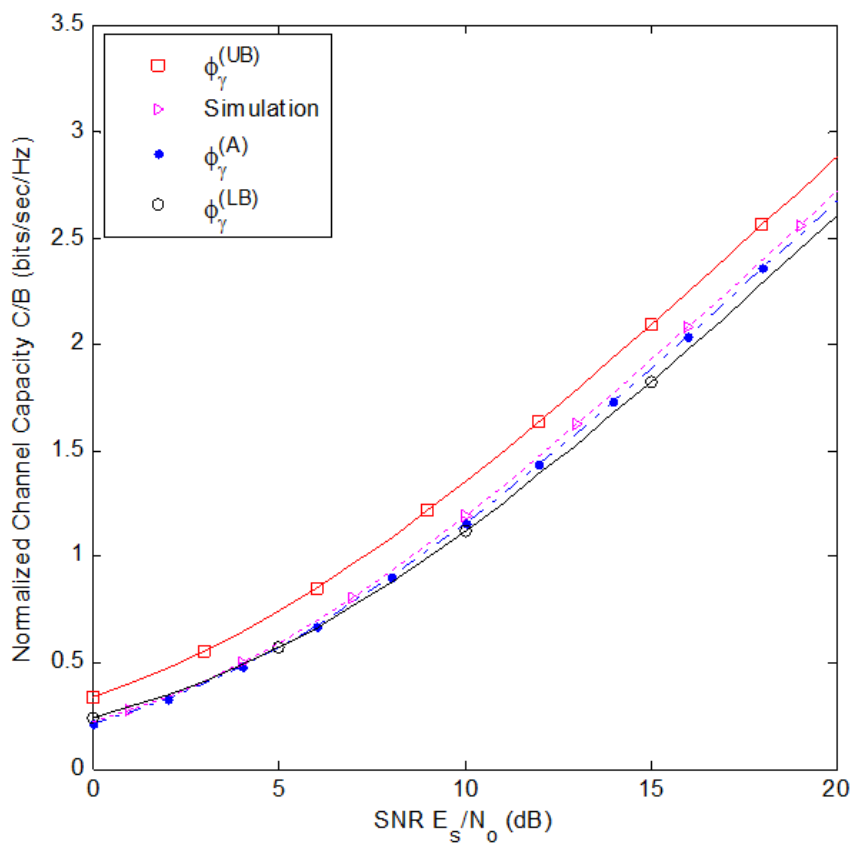

Figure 3. Ergodic capacities of ORA policy in i.n.d Rice fading channels $(K=3)$ with a single cooperative relay $(N=1)$.

\section{Example 2: Rice fading channels}

Consider a CAF relay network with ORA policy in an i.n.d Rice fading channel. It is worth to mention that the exact MGF of harmonic mean SNR for the relayed path presented in [27, Eq. (5) and Table 1] for the Rice fading channel is not tractable as it involves the integral of product of two infinite series (with Bessel function arguments). Moreover, the authors in reference [19] also proposed a general method for ergodic capacity analysis of ORA, OPRA and TCIFR policies. Their expressions are generalized, but the results are limited to only an i.i.d Nakagami-m fading channel, which is obviously due to the non-existence of the closed form MGF expression for the Rice fading channel. Hence, the bounding technique [20] is only available analytical tool for capacity analysis. The MGF for upper bound of the SNR for two-hop relayed path is given by [20]

$$
\phi_{\gamma_{i}}^{(U B)}(s)=\sum_{\substack{k \in\{(s, i),(i, d)\} \\ j \neq k}} \mathrm{~A}_{\mathrm{k}} e^{-K_{k}} 2 I\left[\sqrt{2 A_{j}}, \sqrt{2 K_{j}}, \sqrt{2 A_{k} K_{k}}, 2\left(s+A_{k}\right)\right]
$$

where $A_{i}=1+K_{i} / \Omega_{i}$, and $I[a, b, c, d]=\frac{1}{d} e^{\frac{c^{2}}{2 d}} Q\left(b \sqrt{\frac{d}{\left(d+a^{2}\right)}}, \frac{a c}{\sqrt{d\left(d+a^{2}\right)}}\right)-\frac{a^{2}}{d\left(d+a^{2}\right)} e^{\frac{c^{2}-b^{2} d}{2\left(d+a^{2}\right)}} I_{0}\left(\frac{a b c}{\left(d+a^{2}\right)}\right)$

while the MGF for the lower bound may be computed as $\phi_{\gamma_{i}}^{(L B)}(s)=\phi_{\gamma_{i}}^{(U B)}(s / 2)$. In (16), $K_{k}$ is the Rice fading parameter, $I_{0}($.$) is the zero order modified Bessel function and Q(.$.$) is the first order$ Marcum $Q$-function. Fig. 3 compares approximation and bounds for the ergodic capacity analysis of the CAF relay networks in the Rice fading channels for i.n.d case. The general trend depicted in this figure is similar to those observed for the Rayleigh fading environment in Fig. 2. 


\section{Example 3: Nakagami-m fading channels}

Figures 4, 5, and 6 illustrate the ergodic capacity analysis with ORA, OPRA and TCIFR policies under the i.n.d Nakagami fading channels respectively.

Curves of upper/lower bounds are also generated using [20, eq. (8)] (i.e.,

$$
\phi_{\gamma_{i}}^{(U B)}(s)=\sum_{\substack{k \in\{(s, i),(i, d)\} \\ j \neq k}} \frac{\Gamma\left(m_{k}+m_{j}\right)}{m_{k} \Gamma\left(m_{k}\right) \Gamma\left(m_{j}\right)}\left(\frac{\Omega_{j} m_{k}}{s \Omega_{j} \Omega_{k}+\Omega_{j} m_{k}+\Omega_{k} m_{j}}\right)^{m_{k}}{ }_{2} F_{1}\left(1-m_{j}, m_{k} ; 1+m_{k} ; \frac{\left(s \Omega_{k}+m_{k}\right) \Omega_{j}}{s \Omega_{j} \Omega_{k}+\Omega_{j} m_{k}+\Omega_{k} m_{j}}\right)
$$

where $\Omega_{q}=E\left[\gamma_{q}\right]$ corresponds to the mean SNR of link $q, m_{q}$ is the Nakagami-m fading index and $\Gamma(\cdot)$ is the gamma function) for comparison. It is worth to mention that the exact MGF of the harmonic mean SNR for the relayed path presented in [27, Table 1] for the Nakagami-m channel is not tractable as it involves the product of the Bessel functions with complicated arguments. Hence, the bounding technique [20] is only available analytical tools for capacity analysis. Similar to Figures 2 and 3, in Figures 4-6, we notice that the curve with the approximate MGF method is very close to the exact (simulation) for each of the three adaptive source transmission policies.

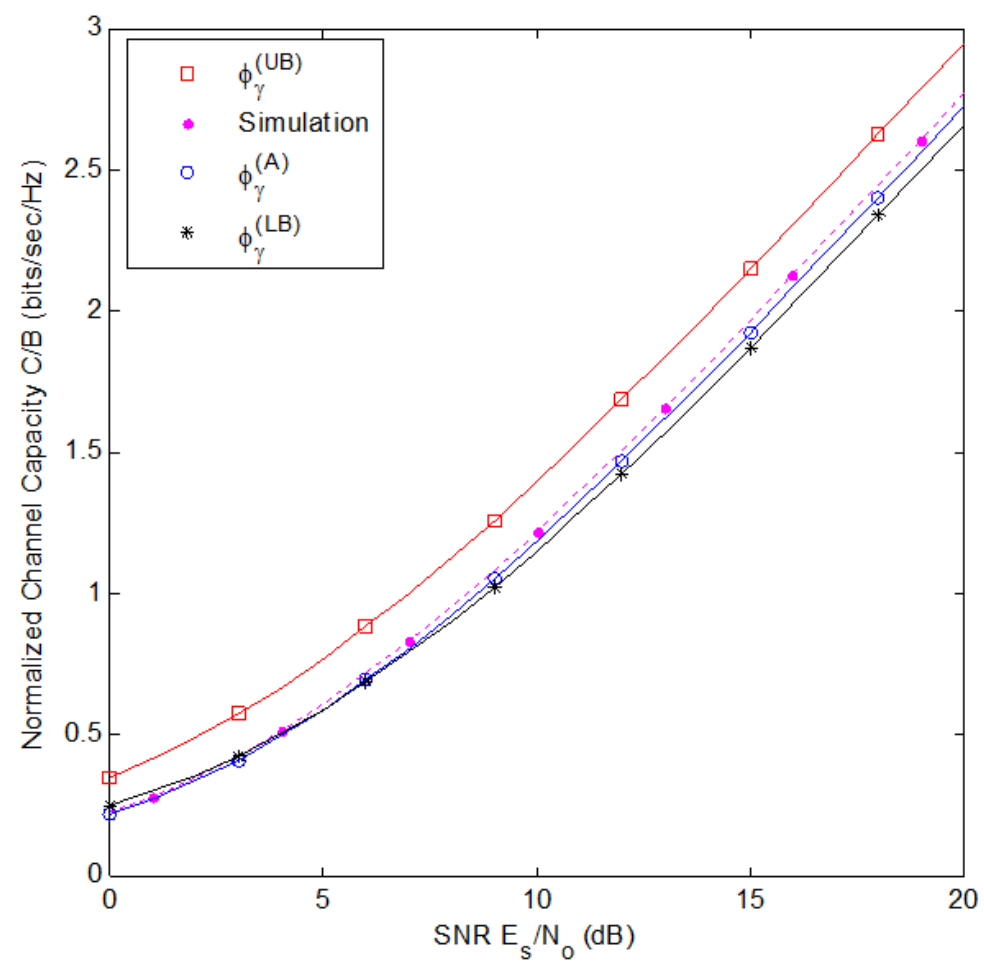

Figure 4. Ergodic capacities of ORA policy in i.n.d Nakagami fading channels $(m=3)$ with a single cooperative relay $(N=1)$. 
International Journal of Wireless \& Mobile Networks (IJWMN) Vol. 8, No. 5, October 2016

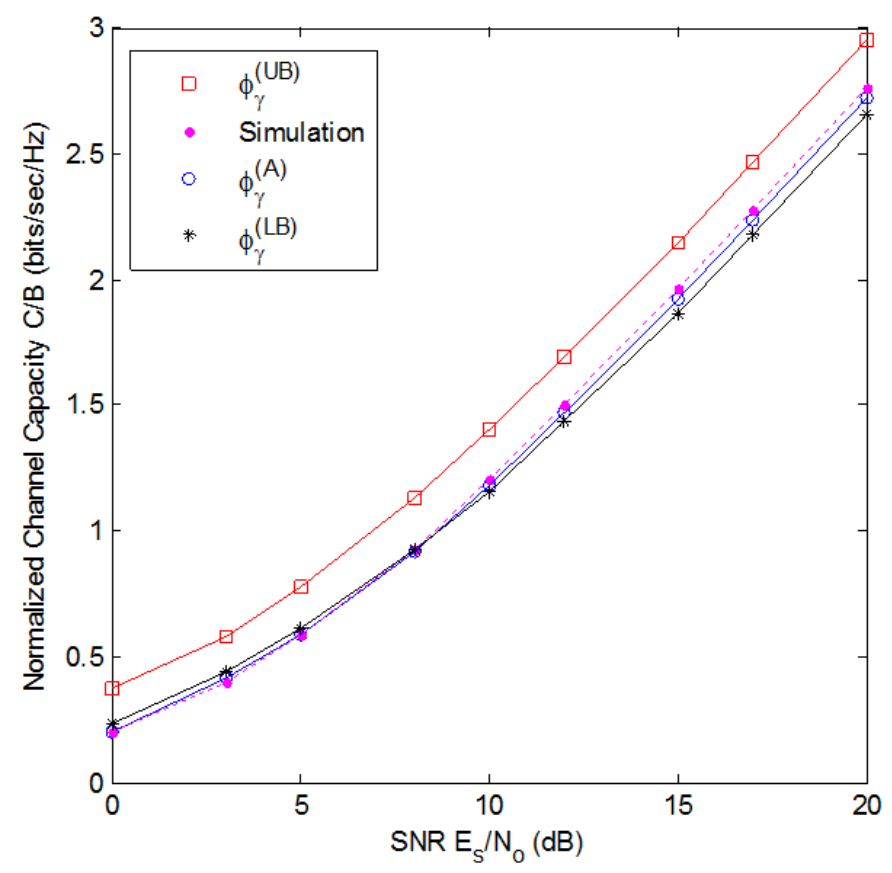

Figure 5. Ergodic capacities of OPRA policy in i.n.d Nakagami fading channels $(m=3)$ with a single cooperative relay $(N=1)$.

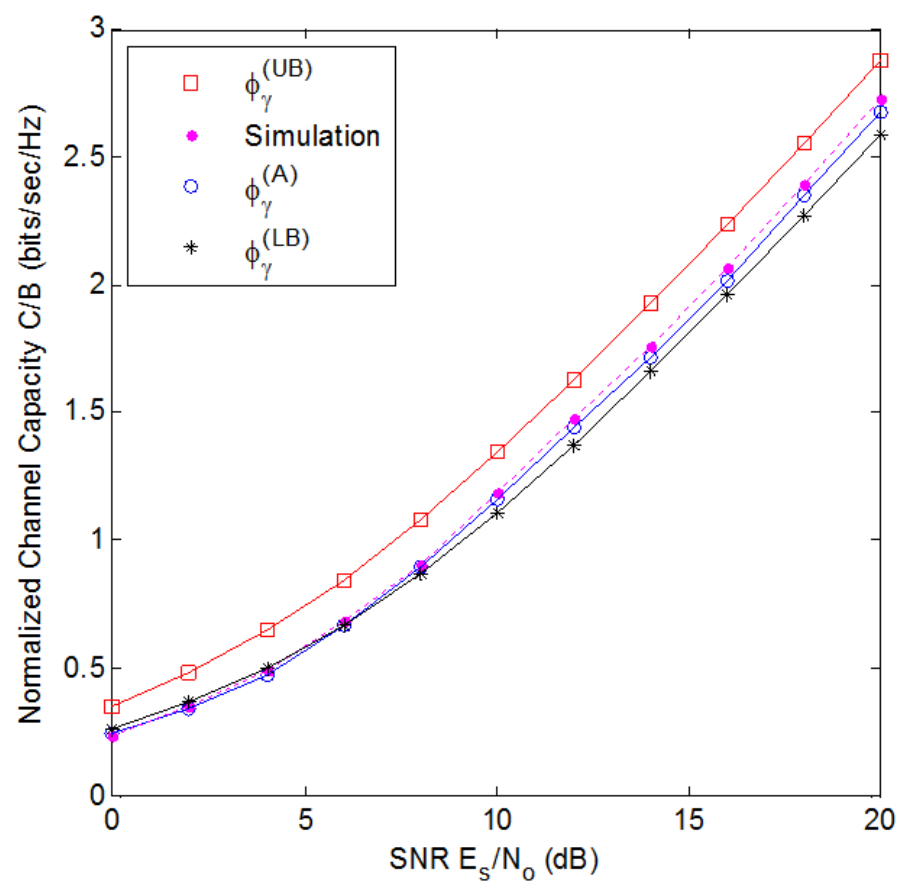

Figure 6. Ergodic capacities of TCIFR policy in i.n.d Nakagami fading channels $(m=3)$ with a single cooperative relay $(N=1)$ 


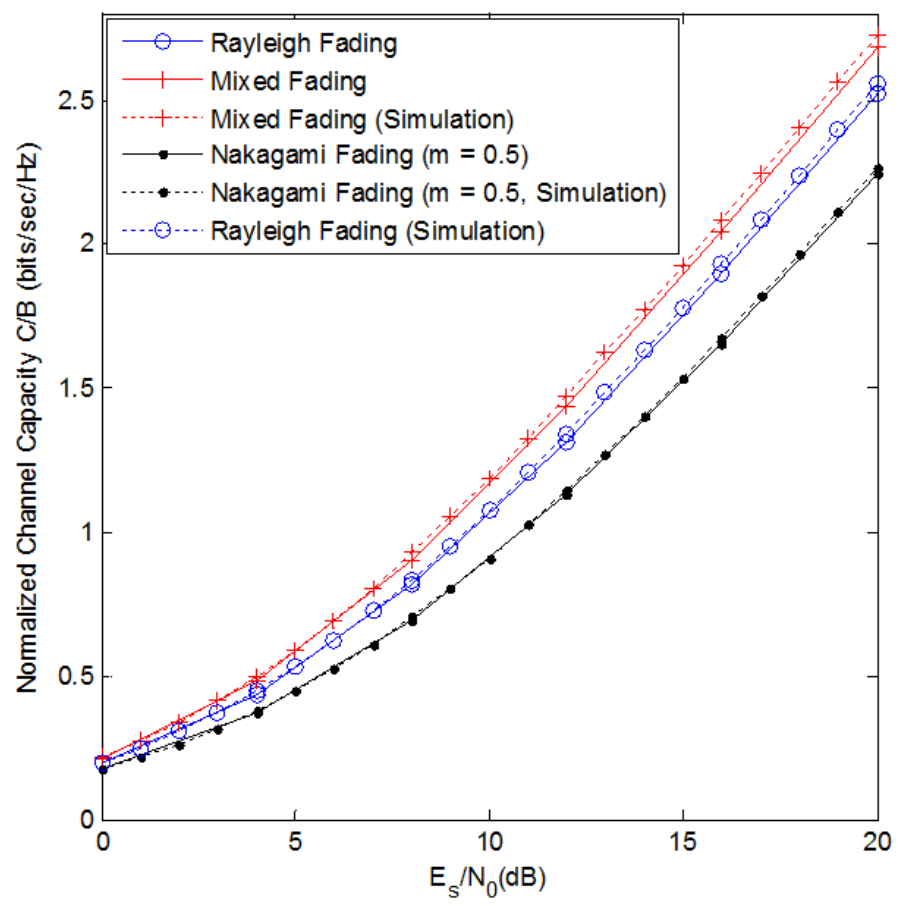

Figure 7: Ergodic capacities of ORA policy under Rayleigh, Nakagami-m and mixed fading environments with a single cooperative relay $(N=1)$.

\section{Example 4: Mixed fading channels}

To show the significant advantage of our framework, which provides generality and flexibility of the approximate MGF $\left(\phi_{\gamma}^{(A)}(s)\right)$, Figure 7 depicts the ergodic capacity analysis of ORA policy under Rayleigh, Nakagami-m and mixed fading environments with single diversity $(\mathrm{N}=1)$. For the mixed fading case, we have chosen Rayleigh fading between S-D link, Rice Fading with $\mathrm{K}=3$ on $S-R_{1}$ link and Nakagami fading with $\mathrm{m}=4$ between $\mathrm{R}_{1}-\mathrm{D}$ link. To show the accuracy of approximate MGF method, corresponding Monte Carlo simulations are also plotted. We observe that in addition to the homogenous fading scenario, our proposed approximate MGF method can also support the mixed fading environments, which cannot be obtained from the exact and bounding techniques in the existing propositions. To the best of our knowledge, the ergodic capacity analysis of CAF relay networks under mixed fading environment with this simplicity has not been considered in the previous literature.

\section{Conclusions}

This paper presents a new accurate approximate MGF method for evaluating the ergodic channel capacities of cooperative non-regenerative multi-relay networks in a myriad of fading environments and under three distinct source-adaptive transmission policies: (i) constant power with optimal rate adaptation (ORA); (ii) optimal joint power and rate adaptation (OPRA); and (iii) fixed rate with truncated channel inversion (TCIFR). The proposed frame work based on the approximate moment generating function (MGF) method bring significant changes in development of capacity system model and is sufficiently general/flexible to encapsulate all 
types of fading environments (especially for the analysis of mixed fading case). In addition to simplifying computation complexity of ergodic capacity for CAF relaying schemes treated in literature, we also derived the closed form expressions of the above three adaptive source transmission policies under Nakagami-m fading with i.n.d statistics. The accuracy of our proposed method has been validated with closed-form formulas that are readily available for specific fading environments in terms of bounds and via Monte Carlo simulations.

\section{APPENDiX A}

In this appendix, we will derive the decomposition of the closed form approximate MGF in (5) that facilitates closed form expression for the ergodic capacity. For instance, the approximate MGF of a single two-hop CAF relayed system is given by

$$
\begin{aligned}
\phi_{\gamma}^{(A)}(s) & \approx \phi_{\gamma_{s, d}}(s)\left(\phi_{\gamma_{s . i}}(s)+\phi_{\gamma_{i . d}}(s)-\phi_{\gamma_{s . i}}(s) \phi_{\gamma_{i, d}}(s)\right) \\
& \approx \phi_{\gamma_{s, d}}(s) \phi_{\gamma_{s . i}}(s)+\phi_{\gamma_{s, d}}(s) \phi_{\gamma_{i . d}}(s)-\phi_{\gamma_{s . d}}(s) \phi_{\gamma_{s . i}}(s) \phi_{\gamma_{i, d}}(s)
\end{aligned}
$$

Substituting the individual MGF expression for the Nakagami-m channel [1, Table 2.2] and applying partial fraction decomposition, we obtain

$$
\begin{aligned}
& \phi_{\gamma}^{(A)}(s) \approx \sum_{p=1}^{m_{s, d}} \frac{\alpha_{p}}{\left(1+\frac{\Omega_{s, d}}{m_{s, d}} s\right)^{p}}+\sum_{q=1}^{m_{s, i}} \frac{\alpha_{q}}{\left(1+\frac{\Omega_{s, i}}{m_{s, i}} s\right)^{q}}+\sum_{r=1}^{m_{s, d}} \frac{\alpha_{r}}{\left(1+\frac{\Omega_{s, d}}{m_{s, d}} s\right)^{r}}+\sum_{w=1}^{m_{i, d}} \frac{\alpha_{w}}{\left(1+\frac{\Omega_{i, d}}{m_{i, d}} s\right)^{w}} \\
& -\left(\sum_{t=1}^{m_{s, d}} \frac{\alpha_{t}}{\left(1+\frac{\Omega_{s, d}}{m_{s, d}} s\right)^{t}}+\sum_{u=1}^{m_{s, i}} \frac{\alpha_{u}}{\left(1+\frac{\Omega_{s, i}}{m_{s, i}} s\right)^{u}}+\sum_{v=1}^{m_{i, d}} \frac{\alpha_{v}}{\left(1+\frac{\Omega_{i, d}}{m_{i, d}} s\right)^{v}}\right) \\
& \text { where, } \alpha_{p}=\frac{\left(-\frac{m_{s, i}}{m_{s, d}} \frac{\Omega_{s, i}}{\Omega_{s, d}}\right)^{m_{s, d}-p}}{\left(1-\frac{\Omega_{s, i}}{\Omega_{s, d}} \frac{m_{s, d}}{m_{s, i}}\right)^{m_{s, d}-p+1}}, \quad \alpha_{q}=\frac{\left(-\frac{m_{s, i}}{m_{s, d}} \frac{\Omega_{s, d}}{\Omega_{s, i}}\right)^{m_{s, i}-q}}{\left(1-\frac{\Omega_{s, d}}{\Omega_{s, i}} \frac{m_{s, i}}{m_{s, d}}\right)^{m_{s, i}-q+1}} \text {, } \\
& \alpha_{r}=\frac{\left(-\frac{m_{i, d}}{m_{s, d}} \frac{\Omega_{i, d}}{\Omega_{s, d}}\right)^{m_{s, d}-r}}{\left(1-\frac{\Omega_{i, d}}{\Omega_{s, d}} \frac{m_{s, d}}{m_{i, d}}\right)^{m_{s, d}-r+1}}, \alpha_{w}=\frac{\left(-\frac{m_{i, d}}{m_{s, d}} \frac{\Omega_{s, d}}{\Omega_{i, d}}\right)^{m_{i, d}-w}}{\left(1-\frac{\Omega_{s, d}}{\Omega_{i, d}} \frac{m_{i, d}}{m_{s, d}}\right)^{m_{i, d}-w+1}}, \\
& \alpha_{t}=\left(\frac{m_{s, d}}{\Omega_{s, d}}\right)^{m_{s, d}-t} \sum_{j=1}^{m_{s, d}-t} \frac{(-1)^{m_{s, d}-t}\left(\frac{\Omega_{s, i}}{m_{s, i}}\right)^{j}}{\left(1-\frac{\Omega_{s, i}}{m_{s, i}} \frac{m_{s, d}}{\Omega_{s, d}}\right)^{j+1}} \frac{\left(\frac{\Omega_{i, d}}{m_{i, d}}\right)^{m_{s, d}-t-j}}{\left(1-\frac{\Omega_{i, d}}{m_{i, d}} \frac{m_{s, d}}{\Omega_{s, d}}\right)^{m_{s, d}-t-j+1}},
\end{aligned}
$$


$\alpha_{u}=\left(\frac{m_{s, i}}{\Omega_{s, i}}\right)^{m_{s, i}-u} \sum_{j=1}^{m_{s, i}-u} \frac{(-1)^{m_{s, i}-u}\left(\frac{\Omega_{s, d}}{m_{s, d}}\right)^{j}}{\left(1-\frac{\Omega_{s, d}}{m_{s, d}} \frac{m_{s, i}}{\Omega_{s, i}}\right)^{j+1} \frac{\left(\frac{\Omega_{i, d}}{m_{i, d}}\right)^{m_{s, i}-u-j}}{\left(1-\frac{\Omega_{i, d}}{m_{i, d}} \frac{m_{s, i}}{\Omega_{s, i}}\right)^{m_{s, i}-u-j+1}},}$
and $\alpha_{v}=\left(\frac{m_{i, d}}{\Omega_{i, d}}\right)^{m_{i, d}-v} \sum_{j=1}^{m_{i, d}-t} \frac{(-1)^{m_{i, d}-v}\left(\frac{\Omega_{s, i}}{m_{s, i}}\right)^{j}}{\left(1-\frac{\Omega_{s, i}}{m_{s, i}} \frac{m_{i, d}}{\Omega_{i, d}}\right)^{j+1}} \frac{\left(\frac{\Omega_{s, d}}{m_{s, d}}\right)^{m_{i, d}-v-j}}{\left(1-\frac{\Omega_{s, d}}{m_{s, d}} \frac{m_{i, d}}{\Omega_{i, d}}\right)^{m_{i, d}-v-j+1}}$

\section{APPENDIX B}

In this appendix, we will derive the closed form expressions for the ergodic capacity with the three distinct adaptive source transmission policies: ORA, OPRA and TCIFR under i.n.d Nakagami-m fading environments.

In order to derive the corresponding PDF of the MGF expression in (A.2), we define a generic MGF function

$$
M(a, k, s)=(1+a s)^{-k}
$$

where $s$ is the Laplace variable.

Taking the inverse Laplace of (B.1), we can easily obtain the PDF in the following form,

$$
f(a, k, \gamma)=\frac{a^{-k}}{\Gamma(k)} \gamma^{k-1} e^{-\gamma / a}
$$

The corresponding complementary CDF (CCDF) using the identity [28, eq. $\left(3.381 .3^{8}\right)$ ] can then be expressed as,

$$
F_{\gamma}^{c}(a, k, \gamma)=\int_{\gamma}^{\infty} f(a, k, \gamma) d t \frac{\Gamma(k, \gamma / a)}{\Gamma(k)}
$$

\section{Optimal Rate Adaptation with Fixed Transmit Power (ORA)}

For instance, the capacity expression of ORA policy with a single relay case is given by [16], [20]

$$
\frac{\bar{C}_{O R A}}{B}=\frac{1}{2} \int_{0}^{\infty} \log _{2}(1+\gamma) f_{\gamma}(\gamma) d \gamma
$$

Using the auxiliary PDF in (B.2), the corresponding ORA capacity can be expressed as 


$$
C_{O R A}(a, k)=\frac{1}{2} \frac{a^{-k}}{\Gamma(k)} \int_{0}^{\infty} \log _{2}(1+\gamma) \gamma^{k-1} e^{-\gamma / a} d \gamma
$$

Now, making use of integral form, $J_{n}(\mu)=\int_{0}^{\infty} t^{n-1} \ln (t+1) e^{-\mu t} d t, \quad \mu>0, n=1,2,3 \ldots . .$. (B.5) can be rewritten as,

$$
C_{O R A}(a, k)=\frac{1}{2} \log _{2}(e) \frac{a^{-k}}{\Gamma(k)} J_{k}(1 / a)
$$

Utilizing the identity $J_{n}(\mu)=\Gamma(n) e^{\mu} \sum_{g=1}^{n} \frac{\Gamma(-n+g, \mu)}{\mu^{g}}[1,15$ B.7], (B.6) can be written as

$$
C_{O R A}(a, k)=\frac{1}{2} e^{-1 / a} \log _{2}(e) \sum_{m=1}^{k} \frac{\Gamma(-m+k, 1 / a)}{a^{k-m}}
$$

The final equation for ergodic capacity of ORA scheme is shown in (8), which can be obtained using the end-to-end PDF obtain via (A.2) with the auxiliary expression in (B.7).

\section{Optimal Power and Rate Adaptation (OPRA)}

Now the ergodic capacity of OPRA policy with a single relay case is given by [16], [20]

$$
\frac{\bar{C}_{O P R A}}{B}=\frac{1}{2} \frac{1}{\ln 2} \int_{\gamma_{0}}^{\infty} \ln \left(\frac{\gamma}{\gamma_{0}}\right) f_{\gamma}(\gamma) d \gamma
$$

Substituting the auxiliary PDF term of (B.2), the corresponding OPRA capacity can be written as

$$
C_{O P R A}(a, k)=\frac{1}{2} \frac{a^{-k}}{\Gamma(k)} \int_{\gamma_{0}}^{\infty} \log _{2}\left(\frac{\gamma}{\gamma_{0}}\right) \gamma^{k-1} e^{-\gamma / a} d \gamma
$$

let $u=\gamma / \gamma_{0}, \quad \gamma_{0} d u=d \gamma$, the expression in (B.9) can be re-written as,

$$
C_{\text {OPRA }}(a, k)=\frac{1}{2} \frac{a^{-k} \gamma_{0}^{k}}{\Gamma(k)} \log _{2}(e) \int_{1}^{\infty} \ln (u) u^{k-1} e^{-u \gamma_{0} / a} d u
$$

Defining an identity $I_{n}(\mu)=\int_{1}^{\infty} t^{n-1} \ln (t) e^{-\mu t} d t, \quad \mu>0, n=1,2,3 \ldots ., \quad[1]$, (B.10) can be written as

$$
C_{O P R A}(a, k)=\frac{1}{2} \frac{a^{-k} \gamma_{0}^{k}}{\Gamma(k)} \log _{2}(e) I_{k}\left(\frac{\gamma_{0}}{a}\right)
$$


Now using the identity $I_{n}(\mu)=\frac{\Gamma(n)}{\mu^{n}} \sum_{d=0}^{n-1} \frac{\Gamma(d, \mu)}{d !}$ from [1, 15A.7], (B.11) can be re-written as

$$
C_{\text {OPRA }}(a, k)=\frac{1}{2} \log _{2}(e) \sum_{m=0}^{k-1} \frac{\Gamma\left(m, \gamma_{0} / a\right)}{m !}
$$

The final equation for ergodic capacity of OPRA scheme is shown in (11), which can be obtained using the end-to-end PDF obtain via (A.2) with the auxiliary expression in (B.12).

\section{Truncated Channel Inversion with Fixed Rate (TCIFR)}

The ergodic capacity of TCIFR policy with a single relay case is given by [20],

$$
\frac{\bar{C}_{T C I F R}}{B}=\frac{1}{2} \log _{2}\left(1+\frac{1}{\int_{\gamma_{0}}^{\infty} \gamma^{-1} f_{\gamma}(\gamma) d \gamma}\right) F_{\gamma}^{c}\left(\gamma_{0}\right)
$$

where $F_{\gamma}^{c}(x)=1-F_{\gamma}(x)=\int_{x}^{\infty} f_{\gamma}(\gamma) d \gamma$ is the complementary CDF of $\gamma$.

Substituting the auxiliary PDF term of (B.2), the integral term in (B.13) can be written as

$$
\int_{\gamma_{0}}^{\infty} \gamma^{-1} f_{\gamma}(\gamma) d \gamma=\frac{a^{-k}}{\Gamma(k)} \int_{\gamma_{0}}^{\infty} \gamma^{k-2} e^{-\gamma / a} d \gamma
$$

Now utilizing identity [25, eq. $\left.\left(3.381 .3^{8}\right)\right]$, (B.14) can be re-written in the following form,

$$
\frac{a^{-k}}{\Gamma(k)} \int_{\gamma_{0}}^{\infty} \gamma^{k-2} e^{-\gamma / a} d \gamma=\frac{\Gamma\left(k-1, \gamma_{0} / a\right)}{a \Gamma(k)}
$$

The final equation for ergodic capacity of TCIFR scheme is shown in section 3.3, which can be obtained using the end-to-end PDF obtained via (A.2) with the auxiliary expression in (B.15).

\section{ACKNOWLEDGEMENT}

This work was supported in part by funding from the Air Force Research Laboratory/Clarkson Aerospace, and the National Science Foundation (0931679 and 1040207).

\section{REFERENCES}

[1] M.K. Simon and M.S. Alouini, (2005) Digital Communication over Fading Channels, New York: Wiley, $2^{\text {nd }}$ edition.

[2] A. J. Goldsmith and P. Varaiya, (1997) "Capacity of Fading Channels with Channel Side Information,” IEEE Trans. Information Theory, vol. 43, pp. 1986-1992. 
[3] N. Laneman, D. Tse, and G. Wornell, (2004) "Cooperative Diversity in Wireless Networks: Efficient Protocols and Outage Behaviour," IEEE Trans. Info. Theory, vol. 50, pp. 3062-3080.

[4] S. Heba, Gami Hiren, M. Mohammad, T. Nazar, M. Edvin, R. Pendse (2000) "High Resolution Joint Time Delay and Frequency Estimation" IEEE Sarnoff Symposium, pp. 1-4.

[5] K. A. Hamdi, (2008) "Capacity of MRC on Correlated Rician Fading Channels," IEEE Trans. Commun., vol. 56, no. 5, pp. 708-711.

[6] R. C. Palat, A. Annamalai and J. H. Reed, (2009) "Accurate Bit Error Rate Analysis of Band limited Cooperative OSTBC Networks under Timing Synchronization Errors," IEEE Trans. Vehic. Tech., vol. 58, pp. 2191-2200.

[7] A. Madsen and J. Zhang (2005) "Capacity Bounds and Power Allocation for Wireless Relay Channels," IEEE Trans. Info. Theory, vol. 51, pp. 2020-2040.

[8] D. Gunduz and E. Erkip, (2007) “Opportunistic Cooperation by Dynamic Resource Allocation," IEEE Trans. Wireless Communications, pp. 1446-1454.

[9] S. Khatalin, (2015) "On the Channel Capacity of SSC diversity system in $\eta-\mu$ and $k-\mu$ fading environments," AEU-International Journal of Electronics and Communications, pp. 1683-1699.

[10] A. Andrawes, (2012) "Performance of Adaptive Modulation with Generalized Selection Combining in Different Practical Scenarios," IEEE International Conference on Computer Engineering \& Systems.

[11] M. Hasna, (2005) "On the capacity of Cooperative Diversity Systems with Adaptive Modulation," Proc. International Conference on Wireless and Optical Communication Networks, pp. 432-436.

[12] R. H. Y. Louie, Y. Li, and B. Vucetic, (2008) "Performance Analysis of Beamforming in Two Hop Amplify and Forward Relay Networks," in Proc. IEEE International Conference on Communications, pp. 4311-4315.

[13] D.Senarante and C. Tellambura, (2010) "Unified Exact Performance Analysis of Two-Hop Amplifyand-Forward Relaying in Nakagami fading,"IEEE Trans. Veh. Tech., vol.59, pp. 1529-1534.

[14] M. Hasna and M. Alouini, (2004) "Harmonic Mean and End-to-End Performance of Transmission System with Relays," IEEE Trans. Communications, vol. 52, no.1, pp. 130-135.

[15] Weifeng, Su. K. S. Ahmed and K. J. Ray Liu, (2008) "Cooperative Communication Protocols in Wireless Networks: Performance Analysis and Optimum Power Allocation," Springer link Wireless Personal Communication, vol. 44 pp. 181-217.

[16] T. Nechiporenko, K. Phan, C. Tellambura and H. Nguyen, (2009) "Capacity of Rayleigh Fading Cooperative Systems under Adaptive Transmission," IEEE Trans. Wireless Comm., vol. 8, pp. 16261631.

[17] S. Ikki and M. H. Ahmed, (2010) “ On the Capacity of Relay-Selection Cooperative-Diversity Networks under Adaptive Transmission,” IEEE Vehicular Technology Conf, pp. 1-5.

[18] P. Kalansuriya, and C. Tellambura, (2009)“Capacity Analysis of a Decode-and-Forward Cooperative Network under Adaptive Transmission,” IEEE CCECE Canadian Conference, pp. 298-303.

[19] M. Di Renzo, F. Graziosi, and F. Santucci, (2010) "Channel Capacity Over Generalized Fading Channels: A Novel MGF-Based Approach for Performance Analysis and Design of Wireless Communication Systems,” IEEE Trans. Vehic. Tech., vol. 59, pp. 127-149.

[20] Bhuvan Modi, A. Annamalai, O. Olabiyi, and R. Chembil Palat, (2012) "Ergodic Capacity Analyses of Cooperative Amplify and Forward Relay Networks over Rice and Nakagami Fading Channels," International Journal of Wireless and Mobile Networks, vol4, no. 1, pp. 97-116.

[21] Bhuvan Modi, O. Olabiyi, A. Annamalai and D. Vaman, (2011) "On Ergodic Capacity of Cooperative Non-Regenerative Relay Networks in Rice Fading Environments," IEEE GLOBECOM Workshop, pp. 348-352.

[22] Bhuvan Modi, A.Annamalai, O. Olabiyi and R.Palat, (2013) "Ergodic Capacity Analysis of Cooperative Amplify-and-Forward Relay Networks over Generalized Fading Channels," Wiley Journal of Wireless Communications and Mobile Computing, pp. 1269-1273.

[23] Bhuvan Modi, A.Annamalai and R.Palat (2011) "An MGF Approach for Performance Evaluation of Non-Regenerative Cooperative Relay Networks with Adaptive Modulation in Nakagami-m Fading Environments," IEEE Wireless Telecommunications Symposium, pp. 1-6.

[24] R. Chembil Palat, A. Annamalai, and J. Reed, (2008) "An Efficient Method for Evaluating Information Outage Probability and Ergodic Capacity of OSTBC Systems," IEEE Comm. Letters, pp. 191-193. 
[25] Bhuvan Modi, O.Olabiyi, A. Annamalai, (2016) "Joint-Design of Link-Adaptive Modulation and Coding with Adaptive ARQ for Cooperative Amplify and Forward Relaying System," International Journal of Computer Networks and Communications, pp. 195-212.

[26] O. Olabiyi, and A. Annamalai, (2011) "ASER Analysis of Cooperative Non-Regenerative Relay Systems over Generalized Fading Channels" IEEE International Conf. on Comp. Commu. and Networks, pp.1-6.

[27] M. Di Renzo, F. Graziosi, and F. Santucci, (2009) "A Unified Framework for Performance Analysis of CSI Assisted Cooperative Communications over Fading Channels," IEEE Trans. Communications, vol. 57, no. 9, pp. 2551-2557.

[28] I. S. Gradshteyn and I. M. Ryzhik, (1995) Table of Integrals, Series and Products, Academic Press.

\section{AUTHORS}

Dr. Bhuvan Modi received PhD. degree from Prairie View A \& M University, Texas A \& M University System, in 2012. He earned his M.S. degree in Electrical Engineering from Lamar University, United States of America, M.S. degree in Electronics and Communication Engineering from Dharmsinh Desai University, India, and the B.S. degree in Electronics and Communication Engineering from North Gujarat University, India, in 2009, 2002 and 2001, respectively. He is currently working as a Senior Member of Technical Staff at AT\&T Mobility Lab Seattle, WA. Currently Dr. Modi serves as an editorial committee//International

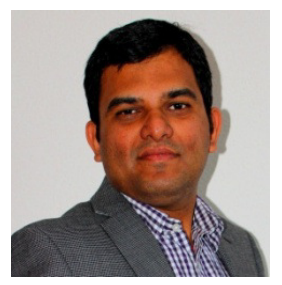
editorial board member for journals and organizations, namely the International Journal of Wireless and Mobile Networks (IJWMN), The Standard International Journals (SIJ), the International Journal of Wireless and Mobile Communication for Industrial Systems, Science \& Engineering Research Support Society, and First International Workshop on Wireless and Mobile Communication for Industrial Systems (WMCIS 2015) and has also been invited to serve on the international editorial board committee member for the Journal of Advanced Research in Wireless, Mobile \& Telecommunication. He received 'Student Travel Grant Award' to present his work at the IEEE MILCOM'11 and achieved excellent work appreciation certificate award from Vice President and CEO, AT\&T Mobility Lab for individual contribution towards successfully launch of WiFi Calling Service in the US Market. Over the last few years, Dr. Modi has published over a dozen peer reviewed conference and journal articles. His current research interests include cross-layer design/optimization for adaptive-link cooperative relay networks, 4G/5G Wireless Technologies, Openstack and software-defined radios.

Dr. Oluwatobi O. Olabiyi received the B.Sc. degree in Electronic and Electrical Engineering from Obafemi Awolowo University, Ile-Ife and M.S. and PhD degrees in Electrical Engineering from Prairie View A\&M University, Texas. Over the last few years, he has co-authored approximately two-dozen peerreviewed conference and journal articles. He was the recipient of the Roy G. Perry College of Engineering Outstanding Masters Student of the Year Award (2011) and the National Society of Black Engineer's Golden Torch Award for Graduate Student of Year (2012). His research interests include dynamic spectrum access, MIMO, cooperative communications, statistical signal processing, compressive sensing, machine-learning and optimization techniques.

Dr. Annamalai is presently the Director of Center of Excellence for Communication Systems Technology Research, a Texas A\&M Board of Regents approved University Research Center at the Prairie View A\&M University, and a tenured faculty member in the Department of Electrical and Computer Engineering. He has over 20 years of research/teaching experience in wireless communications at Motorola, University of Victoria, Air Force Research Laboratory, Virginia Tech and PVAMU with approximately 200 peer-reviewed publications and 5 book chapters. Dr. Annamalai has been honored by his colleagues on numerous occasions for his excellence in research including
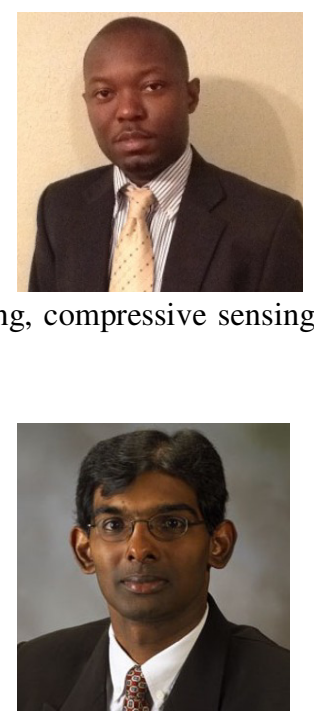
International Journal of Wireless \& Mobile Networks (IJWMN) Vol. 8, No. 5, October 2016

winning the 2011 Roy G. Perry College of Engineering Outstanding Faculty (Research) Award, IEEE Leon Kirchmayer Prize Paper award, ASEE/AFOSR Summer Faculty Fellowships, NSERC Doctoral Prize, CAGS/UMI Distinguished Doctoral Dissertation Award, IEEE VTS/Motorola Daniel E. Noble Fellowship, among others. He had served on the Editorial Boards of four IEEE journals/transactions in the last 12 years, and has helped to organize a few major IEEE conferences on wireless communications including serving in the capacity of Technical Program Chair of the 2002 IEEE Vehicular Technology Conference in Vancouver, Canada. His current research interests include cooperative spectrum sensing, compressive sensing, cross-layer design for scalable multimedia transmission and cooperative wireless communications. 
International Journal of Wireless \& Mobile Networks (IJWMN) Vol. 8, No. 5, October 2016

INTENTIONAL BLANK 